\title{
Mesure résistive des champs de surchauffe et de surrefroidissement sur des films supraconducteurs divisés
}

\author{
C. Rousselle, J. Blot et Y. Pellan \\ Institut National des Sciences Appliquées, BP 14A, 35043 Rennes Cedex, France \\ (Reçu le 16 octobre 1979, rívisć le 10 dícembre 1979, accepté le 20 décembre 1979)
}

\begin{abstract}
Résumé. - Nous présentons une nouvelle structure permettant l'observation des états métastables supraconducteurs dans des films minces d'indium. Le film est partiellement divisé en îlots (carrés de $100 \times 100 \mu \mathrm{m}^{2}$ ) reliés par des ponts d'or; il est continu par ailleurs. Cette structure permet la mesure résistive simultanée du champ critique du film et des champs de surchauffe et de surrefroidissement. Les résultats sont en bon accord avec ceux obtenus par des mesures de susceptibilité.
\end{abstract}

\begin{abstract}
A new structure for observing metastable superconducting states in In thin films is presented. Over a fraction of its length, the film is divided into small islands (squares of $100 \times 100 \mu \mathrm{m}^{2}$ ) connected by normal metal (Au) bridges. Purely resistive measurements allow simultaneous observations to be made of both the superheating and supercooling fields in the islands and the critical field in the continuous region. Results are in good agreement with those obtained from susceptibility measurements.
\end{abstract}

Il est bien connu que les états métastables dans les matériaux supraconducteurs de type I sont aisément observables sur un ensemble de sphères isolées les unes des autres; chaque grain constituant un centre de nucléation indépendant, la transition accidentelle de l'un d'eux ne se propage pas aux autres. Pour les sphères pratiquement parfaites la transition $\mathrm{S} \rightarrow \mathrm{N}$ ou $\mathrm{N} \rightarrow \mathrm{S}$ se produit au champ limite de surchauffe $H_{\mathrm{SH}}(t)$ ou de surrefroidissement $H_{\mathrm{SC}}(t)$. De la même manière les films d'indium à comportement de type $\mathrm{I}$, divisés en milliers de motifs réguliers et régulièrement espacés présentent d'importants retards à la transition sous champ magnétique [1].

La transition d'un ensemble de motifs isolés électriquement les uns des autres est habituellement détectée par des mesures inductives [1-3]. L'objet de cette lettre est de montrer sur des films d'indium que l'on peut, au cours d'une mesure résistive à quatre points, déterminer à la fois le champ critique d'un film et les champs de retard à la transition d'un alignement de motifs isolés les uns des autres au plan des propriétés supraconductrices.

L'échantillon B (Fig. 1) adapté à cette mesure n'est divisé que sur une portion $l$ de sa partie $L$ utile à la mesure : les parties continues doivent transiter au champ critique du film $H_{\mathrm{CF}}$ alors que les petits motifs peuvent être le siège d'états métastables. La continuité électrique entre les motifs d'indium est (a)

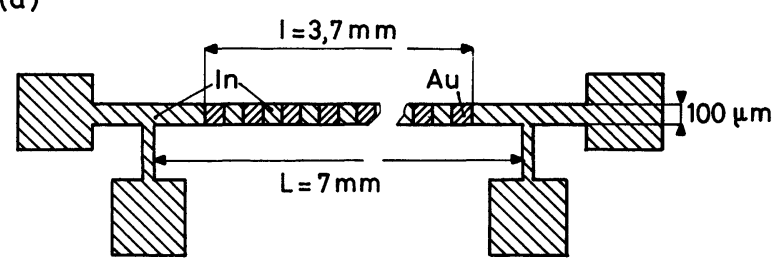

(b)

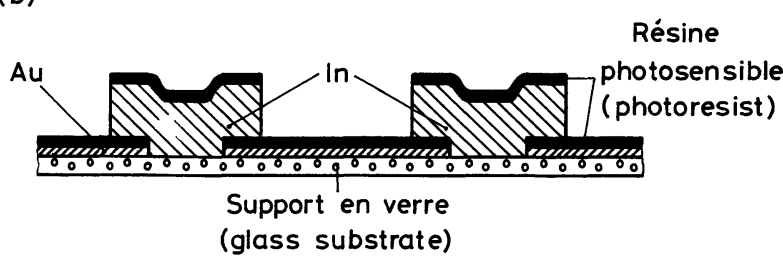

Fig. 1. - a) Géométrie d'un échantillon permettant à la fois la mesure résistive du champ critique et des champs de surchauffe et de surrefroidissement d'un film d'indium; $b$ ) Principe de la réalisation des contacts or-indium.

[a) Sample geometry providing both resistive measurements of superheating and supercooling fields and of the critical field of an indium film ; $b$ ) Schematical representation of gold-indium contacts.]

assurée par des ponts de métal normal, l'or. La réalisation de cet échantillon comporte deux évaporations et plusieurs opérations de photogravure pour 
arriver au schéma détaillé figure 1. La partie divisée comporte 18 pavés de $100 \times 100 \mu \mathrm{m}^{2}$, espacés de $100 \mu \mathrm{m}$. Le contact entre l'or et l'indium est assuré uniquement par la tranche, ce qui limite considérablement les interfaces où peut se développer l'interdiffusion des deux métaux. Les matériaux sont évaporés par effet Joule, sous un vide d'environ $10^{-6}$ torr. Les substrats en verre sont à la température ambiante. La couche d'indium d'épaisseur $d=1,4 \mu \mathrm{m}$ est évaporée simultanément sur les ponts d'or recouverts de résine (cf. Fig. 1) et sur plusieurs lamelles vierges en vue de réaliser des échantillons témoins $A$ et C. L'échantillon A est formé d'un film continu d'indium gravé suivant la géométrie finale présentée par l'échantillon B; il est destiné à l'étude de la transition résistive $\mathrm{du}$ film. L'échantillon $\mathrm{C}$ est constitué d'une rangée de pavés isolés ( 8 de $100 \times 100 \mu \mathrm{m}^{2}$ et deux plus petits aux extrémités), espacés de $100 \mu \mathrm{m}$; il permet l'étude des champs de retard à la transition. La transition de ce type d'échantillon est détectée par la mesure de la variation de fréquence d'un circuit résonnant $L C$ dont l'inductance est formée d'une bobine plate qui entoure l'échantillon [1].

Le dispositif expérimental décrit dans un précédent article [4] permet de relever directement, à toute température, des courbes de transition comme celles de la figure 2 obtenues sous champ magnétique parallèle à la surface de l'échantillon, à $t=0,927$.

Sous champ croissant, l'échantillon non divisé A transite brutalement au champ critique du film $H_{\mathrm{CF} \|}$ déterminé comme indiqué sur la figure $2 a$. Sa valeur dépend de l'épaisseur du film et de la température $[2,4]$. Il est bien connu que de tels échantillons ne peuvent être sujet à la surchauffe. Ils peuvent au contraire présenter un léger retard à la condensation $\mathrm{N} \rightarrow \mathrm{S}$ (Fig. 2a). Nous noterons ce champ de retard $H_{\mathrm{R}}$.

La détection inductive $\Delta v(H)$ de la transition de l'échantillon $\mathrm{C}$ donne des cycles comme celui de la figure $2 c$ où chaque saut correspond à la transition d'un grain (ou de deux). Dans ce type de mesure, les champs de retard $H_{\mathrm{SH}}$ et $H_{\mathrm{SC}}$ sont définis par la transition du dernier motif $[2,5]$.

La transition résistive de l'échantillon B (Fig. 2b) s'effectue en deux étapes bien distinctes, sous champ croissant comme sous champ décroissant. Ce cycle comparé à ceux des figures $2 a$ et $2 c$ est aisément interprétable. Sous champ croissant les parties non divisées de l'échantillon transitent d'un seul bloc au champ critique $H_{\mathrm{CF}}^{\prime}=H_{\mathrm{CF}}$ alors que sous champ décroissant on observe un faible retard à la transition à peine séparé en deux étapes qui correspondent chacune à la transition de l'une des deux parties continues du film (voir Fig. 1). Ce champ de retard $H_{\mathrm{R}}^{\prime}$ est très comparable au champ $H_{\mathrm{R}}$ observé sur l'échantillon A (Fig. 2a). Etant donné la faible variation de résistance $(\Delta R(H)=45 \mathrm{~m} \Omega)$ enregistrée lors de la transition complète de l'échantillon et compte tenu du rapport signal sur bruit de notre système de mesure

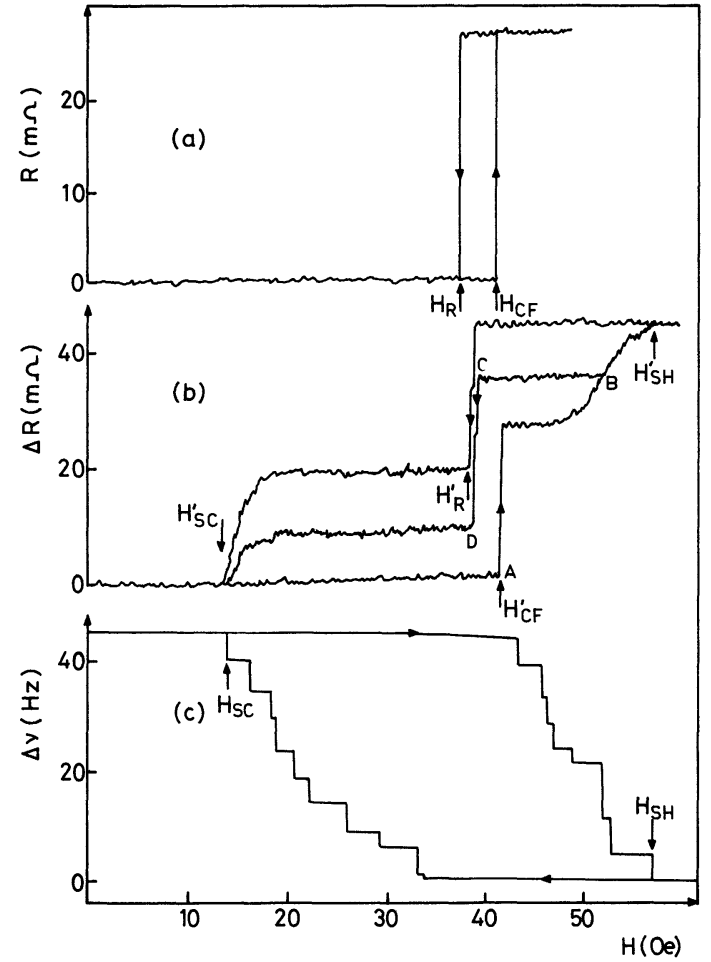

Fig. 2. - Transition sous champ magnétique parallèle d'échantillons d'épaisseur $1,4 \mu \mathrm{m}$, à la température réduite $t=0,927$. a) Mesure résistive du champ critique sur le film $\mathrm{A}$, non divisé; b) Mesure résistive des champs de transition sur l'échantillon B (voir Fig. 1); c) Mesure inductive des champs de surchauffe et de surrefroidissement sur un échantillon divisé $C$.

[Transition under parallel magnetic field of samples $1.4 \mu \mathrm{m}$ thick, at reduced temperature $t=0.927$. a) Resistive measurement of critical field of the A individed film; $b$ ) Resistive measurements of transition fields of the B sample (Fig. 1);c) Inductive measurements of superheating and supercooling fields for the $\mathrm{C}$ divided film.]

résistive par détection synchrone, on ne peut visualiser la transition grain par grain des 18 motifs d'indium qui forment la partie centrale de l'échantillon. Il n'y a cependant aucune ambiguïté sur le sens de la mesure : les variations progressives de résistance enregistrées aux extrémités du cycle figure $2 b$ caractérisent bien le comportement d'un ensemble de grains qui transitent les uns après les autres suivant qu'ils renferment plus ou moins de défauts $[2,5]$. Le balayage $0 \mathrm{ABCD} 0$ montre que les motifs qui présentent la plus mauvaise surchauffe ont également tendance à transiter plus tôt sous champ décroissant. Les champs $H_{\mathrm{SH}}^{\prime}$ et $H_{\mathrm{SC}}^{\prime}$ sont déterminés par les extrémités du cycle comme indiqué sur la figure $2 b$.

Nous avons montré [4] que nos systèmes de mesure (inductive et résistive) donnent les mêmes champs critiques parallèles. De même, nous avons vérifié que sur l'échantillon interconnecté $\mathrm{B}$, ces deux systèmes donnent des courbes de transition semblables : même étalement, même champ critique et mêmes champs limites. La différence des largeurs de transition entre les alignements $B$ et $C$ n'est donc pas imputable à l'utilisation de systèmes de mesure 
différents. Les transitions plus étalées sur l'échantillon $C$ que sur l'échantillon $B$ révèlent seulement que le premier contient des pavés avec des défauts plus importants que ceux existant dans le second; le fait que certains pavés de l'échantillon $C$ ne surchauffent pratiquement pas est concluant à cet égard.

Les deux alignements $B$ et $C$ formés de motifs également espacés et de même taille donnent néanmoins les mêmes champs limites $H_{\mathrm{SH}}$ et $H_{\mathrm{SC}}$ : c'est qu'ils contiennent des pavés de même perfection au sens de l'observation des états métastables et que les ponts de métal normal dans la structure B ne modifient pas ces champs, au moins à cette température. Les mesures ont été effectuées à différentes températures sur les trois types d'échantillons; les champs obtenus sont présentés figure 3 en fonction de la température réduite $t=T / T_{\mathrm{c}}$ où la température critique $T_{\mathrm{c}}=3,395 \mathrm{~K}$ est la même pour les trois échantillons. Il apparaît clairement que la structure réalisée permet à toute température de mesurer le champ critique d'un film et de mettre en évidence les retards à la transition d'un ensemble de motifs ayant la même épaisseur. Dans un large voisinage de $T_{\mathrm{c}}$, les champs de surchauffe $H_{\mathrm{SH}}^{\prime}(t)$ et de surrefroidissement $H_{\mathrm{SC}}^{\prime}(t)$ sont en excellent accord avec ceux $\left(H_{\mathrm{SH}}(t)\right.$ et $\left.H_{\mathrm{SC}}(t)\right)$ déterminés sur un ensemble de grains isolés, mais ils s'en éloignent pour $t<0,7$. A basse température, le contact or-indium peut être la cause d'une germination accidentelle de la phase stable. Ce contact formé par la mise en série de couches minces évaporées qui sont généralement polycristallines $[6,7]$ est certainement complexe. La diffusion conduit en effet soit à la formation de composés substitutionnels du type AuIn, AuIn $2, \ldots$ [8] non supraconducteurs aux températures de nos expériences [9], soit à la formation de composés interstitiels [10]. Ces composés peuvent modifier localement les propriétés de l'indium supraconducteur. Par ailleurs une implantation possible d'argon au moment de la gravure des ponts d'or et l'adsorption lors de la remise à l'air qui précède l'évaporation de la couche d'indium peuvent contaminer le contact. L'ensemble de ces impuretés constitue des sites privilégiés pour la nucléation de la phase stable; leur rôle dans la transition dépend de leur taille par rapport à la longueur de cohérence supraconductrice $\xi(t)$. Près de $T_{\mathrm{c}}$ où $\xi(t)$ est grand, ces centres peuvent n'avoir aucune action, par contre $\xi(t)$ diminuant avec la température ils peuvent engendrer une nucléation accidentelle à basse température. Le paramètre d'ordre dans le supraconducteur est forcément perturbé au voisinage du contact ; cependant la nucléation n'est sans doute pas la conséquence d'un éventuel effet de proximité qui devrait défavoriser la surchauffe [11] tout en allant dans le sens d'un meilleur surrefroidissement [12]. Ceci est contraire à l'expérience et l'on sait d'ailleurs qu'étant donnée la grande solubilité relative de l'or et de l'indium [10], ce couple ne se prête pas à l'observation d'un bon effet de proximité [13].

En conclusion, on peut effectuer une mesure résistive des champs de retard à la transition d'un film supraconducteur en reliant des motifs d'un matériau $S$ par des ponts de métal normal $N$. L'influence du métal $\mathrm{N}$ sur le supraconducteur est minimisée en donnant la plus petite surface possible au contact et en utilisant des motifs $\mathrm{S}$ et $\mathrm{N}$ dont les dimensions sont grandes vis-à-vis des paramètres de diffusion et des longueurs supraconductrices $\lambda(t)$ et $\xi(t)$. Dans

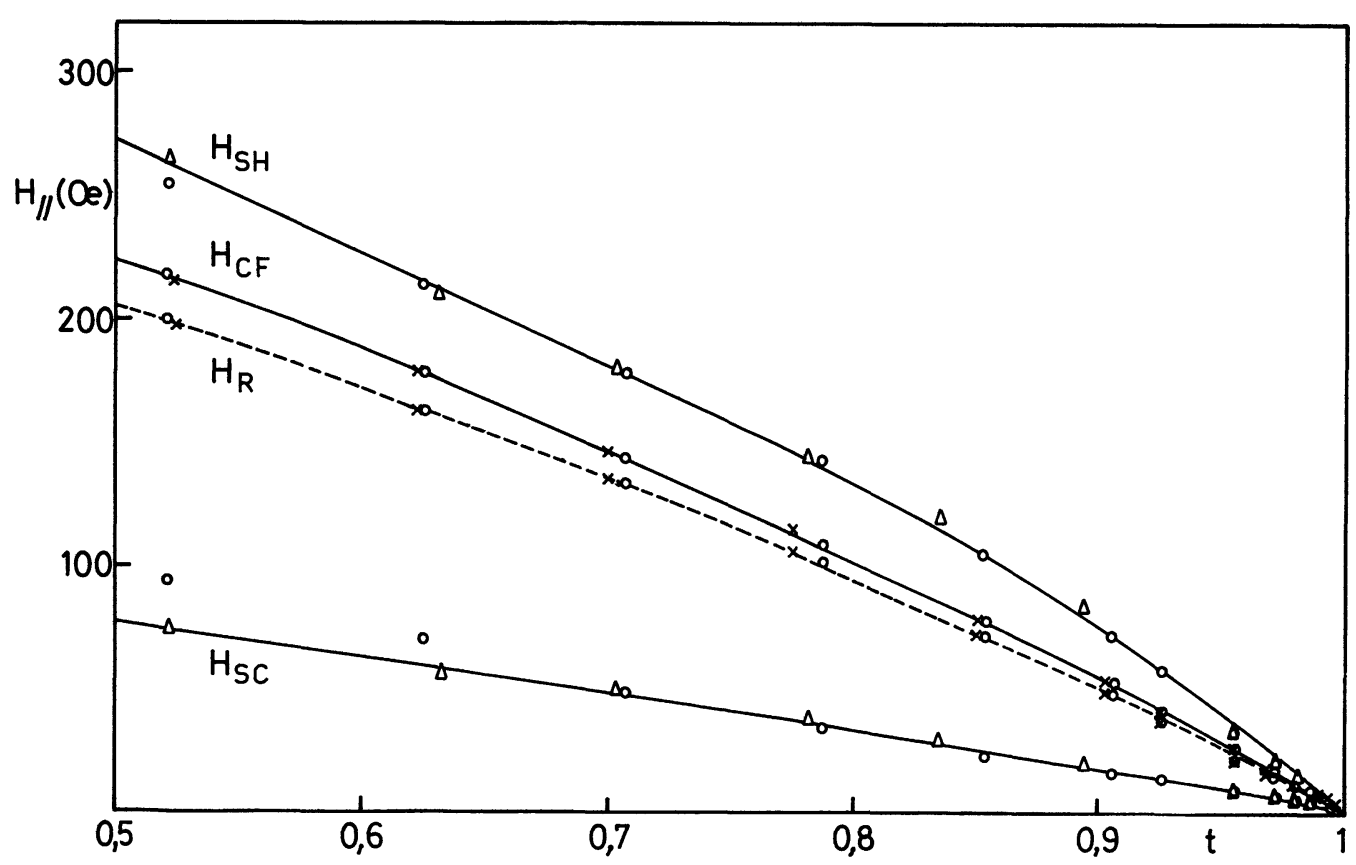

Fig. 3. - Champs parallèles de transition en fonction de la température réduite : $(x)$ échantillon $A,(O)$ échantillon $B,(\triangle)$ échantillon $C$.

[Parallel transition fields as a function of reduced temperature : $(\times)$ A sample, $(O) \mathrm{B}$ sample, $(\triangle) \mathrm{C}$ sample.] 
une large gamme de températures à partir de $T_{\mathrm{c}}$ les ponts de métal normal ne modifient pas les propriétés de l'échantillon puisque les champs limites obtenus sont en excellent accord avec ceux mesurés sur un alignement de motifs isolés les uns des autres. La structure réalisée permet donc l'accès au paramètre de G.L. du film. Les mesures sous champ perpendiculaire qui n'ont pas été présentées ici donnent également des résultats très cohérents. Cette méthode a l'avantage de permettre la comparaison immédiate du champ critique du film à ses champs de retard à la transition.

\section{Bibliographie}

[1] Pellan, Y., Blot, J., Pineau, J. C. and Rosenblatt, J., Phys. Lett. 44A (1973) 415.

[2] Feder, J. and Mclachlan, D. S., Phys. Rev. 177 (1969) 763.

[3] Smith, F. W., Baratoff, A. and Cardona, M., Phys. Kondens. Mater. 12 (1970) 145.

[4] Blot, J., Pellan, Y. and Rosenblatt, J., J. Low Temp. Phys. 30 (1978) 669.

[5] Blot, J., Thèse B.244-158 (1972), Rennes.

[6] Pellan, Y. et Blot, J., à paraître.

[7] Larson, D. C., Physics of Thin Films (Acad. Press Inc. New York) vol. 6, 1971, p. 81.
[8] Simic, V. and Marinkovic, Z., Thin Solid Films 41 (1977) 57.

[9] RoBerts, B. W., Superconductive Materials and some of their properties (U.S. Department of Commerce N.B.S. Technical note 482) 1969.

[10] Anthony, T. R. and Turnbull, D., Phys. Rev. 151 (1966) 495.

[11] Orsay Group on Superconductivity in Quantum Fluids, D.F. Brewer, Ed. (North Holland) 1966.

[12] Hurault, J. P., Phys. Lett. 20 (1966) 587.

[13] Gilabert, A., Ann. Phys. 2 (1977) 203.

Clarke, J., J. Physique Colloq. 29 (1968) C2-29. 